\title{
THE GEOMETRIC AND MATERIAL NONLINEAR ANALYSIS OF CYLINDRICAL SHELLS BY RISE SPAN RATIO
}

\author{
Prof Sangeul Han ${ }^{1}$, Kitae Kim ${ }^{2}$, Dajin $\mathrm{Kim}^{3}$ and Prof Kanggeun Park ${ }^{4}$
}

Abstract- This paper investigates the mechanical characteristics of a combined geometric and material behavior of cylindrical roofs subjected to a concentrated load. The shell are modeled by 3-D general shell element and 3-D composite shell element in the NISA 2016 software. The 3-D shell element includes deformation due to membrane, bending, membrane-bending coupling and transverse shear effects and is suited for modeling moderately thin or thick general shells and laminated composite shells. The 3-D composite shell element consists of a number of layers of perfectly bonded orthotropic and anisotropic materials. The objective of this research is to analyze the load deflection curves considering the combined geometric and material nonlinearity of cylindrical shells and the snap-through phenomenon in the analytical results will be investigated.

Keywords - Mechanical Characteristics, Geometric and Material Behavior, Cylindrical Roofs, Load Deflection Curves

\section{INTRODUCTION}

Cylindrical shells have the structural advantage of a cylindrical curve, for this reason, cylindrical shells are being used widely as structural members in a variety of areas including aircrafts, ships, machines, sports equipment, construction materials, and buildings [1]. Cylindrical shells exhibit various mechanical behaviors according to the shape, boundary condition, and curve. In particular, the behavior characteristics become very sensitive when the shell is thin and row rise span ratio. Unlike plates, the geometry of shell members is composed of surfaces with a limited radius of curvature and the shapes of the surface are diverse. If the span-rise ratio of shell is small, snap-back or snap-through phenomenon can happen on the load-displacement curve. The materials are classified into isotropic, orthotropic, and anisotropic materials according to the material characteristics. Elastic materials are assumed to behave elastically until a certain stress limit, after which combined elastic and plastic behaviors occur. To determine the plastic behaviors, the nonlinear analysis should be performed taking into account the nonlinearity of the material considering plastic theory. Furthermore, geometric nonlinearity should be considered if the deformation is large [2].

Nonlinear analysis methods are classified with geometric nonlinear analysis, material nonlinear analysis, combined geometric and material nonlinear analysis, and boundary nonlinear analysis. This study aims to investigate the combined geometric and material behavior of a flat cylindrical shell. Thus, the mechanical characteristics of geometric and material nonlinearity are analyzed through an analysis of gradually increasing displacement. First of all, the theories of isotropic and anisotropic materials are discussed, and the analytical method for elastoplastic finite element analysis is formulated. Then, the yield theory and hardening effects of materials applied for material nonlinear analysis is defined. The analysis parameters are material thickness, elastic modulus, and hardening effect, and the effects of these parameters on the mechanical characteristics of the geometric and material nonlinear behaviors are analyzed.

\section{PLACTIC THEORY AND NONLINEAR ANALYSIS METHOD \\ A. Plastic Theory \\ 1) General Plasticity Theory-}

An elastic material is assumed to behave elastically until a certain stress limit, after which the combined elastic and plastic behavior occurs. The yield criterion is the relationship that defines the limit of elasticity and the beginning of plasticity under all possible stress conditions. The Tresca yield criterion is a maximum shear stress criterion. For design purposes, if no plastic yielding is to be permitted, the Tresca criterion is more conservative than the von Mises criterion. The maximum difference between these two criteria is $15 \%$ and it occurs in pure shear condition. For most metals, the von Mises criterion is closer to the experimental data than the Tresca criterion. The two-dimensional plane of the von Mises and Tresca yield criteria is shown in Figure 1 [2]. The hardening rule determines how the yield function changes during plastic deformation. Strain hardening can

\footnotetext{
${ }^{1}$ Department of Architectural Engineering, Inha University, Incheon, South Korea

${ }^{2}$ Department of Architectural Engineering, Inha University, Incheon, South Korea

${ }^{3}$ Department of Architectural Engineering, Inha University, Incheon, South Korea

${ }^{4}$ Department of Architectural Engineering, I'ST Institute of Technology, Seoul, South Korea
} 
be explained as follows. A permanent deformation called plastic deformation of a material such as metal under an external load is caused by sliding called the transfer of crystals in the metal. To generate additional plastic deformation, a greater load than before is required. This is called strain hardening, which ultimately increases the stiffness of material. The stress-strain diagram of material which represents strain hardening is expressed as a convex curve, rather than a straight line with a constant slope, in the plastic area. As the strain increases, the stiffness of the material decreases, which is called strain softening [3].

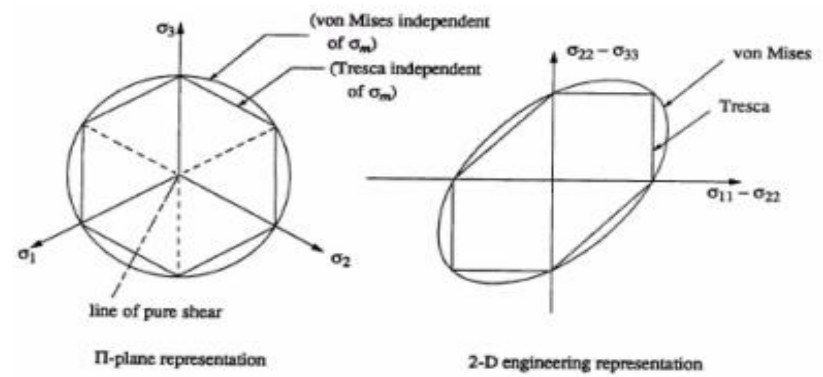

Figure 1. Von Mises anf Treca yield criteria

The magnitude of a stress that causes a permanent deformation or plastic deformation under an external load is called yield stress. If hardening of the material occurs, this yield surface expands and the yield stress increases. The expansion types of yield surface can be classified into an expansion of the same magnitude in all directions, an expansion of a different magnitude in each direction, and a movement of the center while the size of the surface is constant. This is called kinematic hardening. The perfect plasticity theory is the simplest possible assumption and assumes that the yield surface is constant and does not change by plastic deformation. The general behavior of a perfect plastic material is that the increment of plastic strain is not fully determined by stress condition. This is because the flow rule is continuously satisfied when the plastic strain component is multiplied by a coefficient. The perfect plasticity assumption can cause a convergence problem in analysis. This convergence problem can be reduced if the problem is converted to a displacement control situation or a small hardening coefficient is introduced [3].

2) Hill Yield Condition for Anisotropic Elastoplastic Model[4]-

The yield condition determines the stress level when plastic deformation starts based on Eq. (2.1):

$2 f\left(\sigma_{i j}\right)=F\left(\sigma_{22}-\sigma_{a \mathrm{a}}\right)^{2}+G\left(\sigma_{\mathrm{ag}}-\sigma_{11}\right)^{2}+H\left(\sigma_{11}-\sigma_{22}\right)^{2}+2 L \sigma_{2 \mathrm{a}}^{2}+2 M \sigma_{2 \mathrm{a}}^{2}+2 N \sigma_{12}^{2}-1=0$

Where, $F, G, \mathrm{H}, L, M$, and $N$ are the current state characteristic parameters of anisotropy, and 1, 2, and 3 are the three major directions. Finally, the Hill anisotropic yield conditional expression is Eq. (2.2):

$3 f(\sigma)=M_{i j} \sigma_{i} \sigma_{j}-L_{i} \sigma_{i}-K=0$

Where $M_{i j}$ and $L_{i}$ are the hardening parameters related to the effective size of yield surface as well as to the difference in the strength of tension and compression directions according to the direction and deformation of tensile strength, and $\sigma_{\mathrm{i}}$ is the vector form of the stress tensor $\sigma$.

3) Gotoh Yield Condition for Isotropic Elastoplastic Model[5]-

Gotoh pointed out the irrationality of Hill's second-order yield condition for an orthogonal problem. Hill's second-order yield condition expression coincides well with the experimental value or the in-plane distribution of the yield stress shows differences from the experiment results, Hill proposed a yield condition expression of a planar anisotropic material as shown in Eq. (2.3).

$\sigma_{Y}^{4}=A_{1} \sigma_{x}^{4}+A_{2} \sigma_{x}^{a} \sigma_{y}+A_{a} \sigma_{x}^{2} \sigma_{y}^{2}+A_{4} \sigma_{x} \sigma_{y}^{a}+A_{5} \sigma_{y}^{4}+\left(A_{6} \sigma_{x}^{2}+A_{7} \sigma_{x} \sigma_{y}+A_{g} \sigma_{y}^{2}\right) \tau_{x y}^{2}+A_{9} \tau_{x y}^{4}$

\section{B. Nonlinear Analysis of Elastoplastic Large Deformation-}

1) Elastoplastic Material[5,6]-

Eq. (2.4) shows a constitutive equation of the strain increment $d \epsilon_{i j}$ and the stress increment $d \sigma_{i j}$ of a work-hardening elastoplastic material where the hardening parameter $k$ is given as a plastic work $W^{p}$.

$\mathrm{d \epsilon}_{\mathrm{i} j}=d \epsilon_{\mathrm{ij}}^{e}+d \mathrm{\epsilon}_{\mathrm{i} j}^{\mathrm{p}}=\frac{d \sigma_{i j}{ }^{-}}{2 G}+\frac{1-2 v}{E} d \sigma_{m} \delta_{i j}+h \frac{\partial f}{\partial \sigma_{i j}{ }^{2}} \frac{\partial f}{\partial \sigma_{m n}} d \sigma_{m n}=$ 
Eq. (2.5) is the matrix of the reverse relation of Eq. (2.4).

$d \sigma=\left[D^{Q p}\right] d \epsilon=\left(\left[D^{\varepsilon}\right]-\frac{\left[D^{Q}\right]\left[\frac{\partial f}{\partial \sigma}\right]\left[\frac{\partial f}{\partial \sigma}\right]^{T}\left[D^{\alpha}\right]}{\frac{1}{h}+\left(\frac{\partial f}{\partial \sigma}\right)^{T}\left[D^{\alpha}\right]\left[\frac{\partial f}{\partial \sigma}\right]}\right)\{d \epsilon\}$

Here, $\left[D^{\otimes}\right]$ is called elastoplastic modulus matrix.

2) Stiffness Equation[5]-

In the case of an elastoplastic material that exhibits nonlinear behavior, the relation between the nodal force and the nodal displacement is expressed using the principle of the incremental virtual work as Eq. (2.6).

$\int_{V}\{d \sigma\}^{T}\{\delta(d \epsilon)\} d V=\int_{S}\{d T\}^{T}\{\delta(d u)\} d S+\int_{V}\{d F\}^{T}\{\delta(d u)\} d V$

The material is discretized into finite elements, which is simplified by the incremental relationship of displacement-strain into Eq. (2.7):

$d \epsilon=[A] d u=[A][N] d u^{e}=[B] d u^{\theta}$

From the fact that the nodal displacement is unrelated to volume integral, this can be expressed as Eq. (2.8).

$\int_{V}\{d \sigma\}^{T}\{\delta(d \epsilon)\} d V=\int_{V_{Q}}\left(\left[D^{\varepsilon p}\right][B]\left\{d u^{\theta}\right\}\right)^{T}[B] \delta\left(d u^{\theta}\right) d V=\left\{d u^{\theta}\right\}^{T} \int_{V_{Q}}[B]^{T}\left[D^{\varepsilon p}\right][B] d V \delta\left(d u^{\theta}\right)$

We finally get Eq. (2.9) by the principle of incremental virtual work:

$\left[K^{\theta}\right]\left\{d u^{\theta}\right\}=\left\{d F_{5}^{\varepsilon}\right\}+\left\{d F_{V}^{e}\right\}$

$\left[K^{\varepsilon}\right]=\int_{V_{s}}[B]^{T}\left[D^{\varepsilon p}\right][B] d V,\left\{d F_{S}^{\varepsilon}\right\}=\int_{S_{q}}[N]^{T}\{d T\} d S,\left\{d F_{V}^{\varepsilon}\right\}=\int_{V_{\varepsilon}}[N]^{T}\{d F\} d V$

In Eq. (2.10), the matrix $\left[K^{\varepsilon}\right]$ is called the element stiffness matrix, the known nodal force increment is $\mathrm{d} F_{g a}$, the unknown nodal force increment is $\left\{d F_{a b}\right\}$, the known nodal displacement increment is $\left\{d u_{a}\right\}$, and the column matrix of unknown nodal displacement increment is $\left\{d u_{b}\right\}$.

III. NONLINEAR ANALYSIS OF CYLINDRICAL SHELL

A. Geometric and Material Nonlinear Analysis of Shallow Cylindrical Shells-

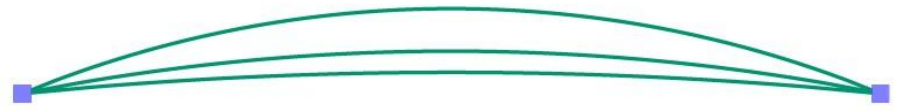

Figure 2. The curve of cylinder

The model is a cylindrical roof with three typed curves. It was assumed that the concentrated load acted on the center of the shell and the nonlinear analysis was performed with a displacement increment at this point. The radius and width of the cylindrical shell are $2540 \mathrm{~mm}$ and $508 \mathrm{~mm}$, respectively, and the elastic modulus is of $E x=42000 \mathrm{MPa}$ and $E y=21000 \mathrm{MPa}$. Initial yield stress is $4.1 \mathrm{MPa}$ in the material direction. Initial shear stress is $2.367 \mathrm{MPa}$ and Work hardening parameter is applied to 0 . The cylindrical roof shell in Figure 2 was modeled as 6 × 6 using 3-D shell elements. For boundary conditions, the hinges of two edges are applied. 3-D 8-node shell elements were used. The number of displacement increments is 30 equal parts. The maximum number allowed for each increment is 100 and the applied tolerance is 0.0001 . The thickness is $21 \mathrm{~mm}$. Figure 3, 4 and 5 represent the load displacement curves for the span rise ratio $0.025,0.05$ and 0.1 . Figure 6 shows a comparison of the curves in Figure 3, 4 and 5.

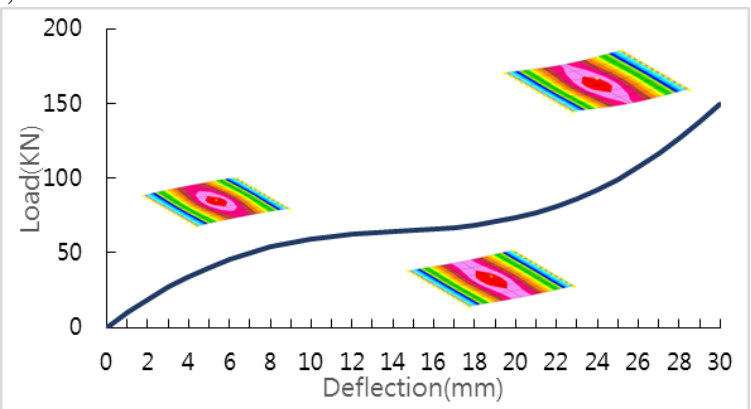

Figure 3. Load displacement curve $($ Span rise ratio $=0.025)$ 


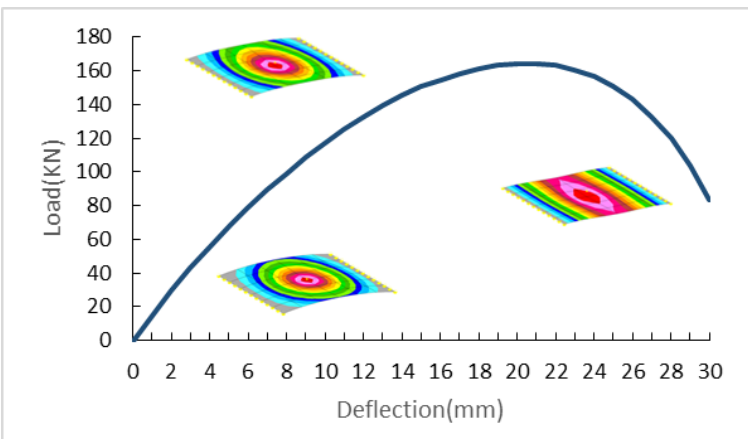

Figure 4. Load displacement curve (Span rise ratio $=0.05)$

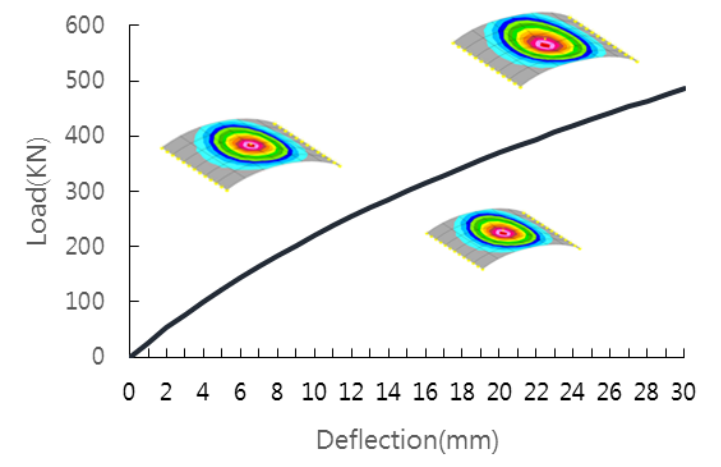

Figure 5. Load displacement curve (Span rise ratio $=0.1)$

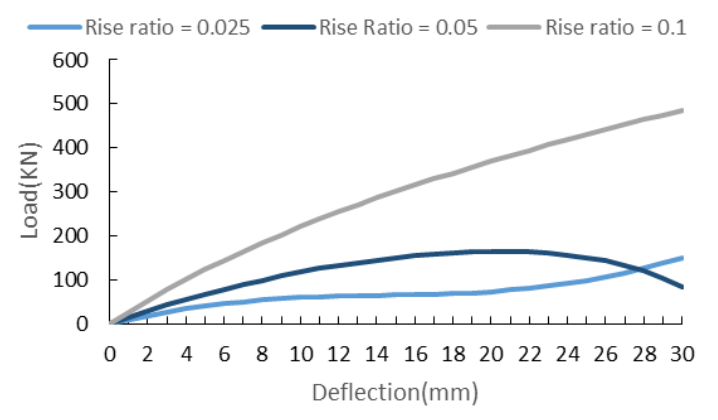

Figure 6. Load displacement curves (Span rise ratio $=0.025,0.05,0.1$ )

B. Geometry and material nonlinear analysis of cylindrical shell-

The geometric and material nonlinearities of the cylindrical shell that subjected to a concentrated load at the center were considered simultaneously for this analysis. The cylindrical roof shell was modeled as 6 x 6 using 8-node 3-D shell elements. For boundary conditions, the hinge points of four edges are applied. For these elements, 3-D 8-node shell elements were used. The number of displacement increments is 30 equal parts. The maximum number of repetitions allowed for each increment is 100 and the applied error is 0.0001 . The thickness is $70 \mathrm{~mm}$. Figure 8,9 and 10 show the load-displacement curves for span rise ratio of $0.2,0.1$, and 0.05 and the elastic moduli of $E x=42000 \mathrm{MPa}$ and $\mathrm{Ey}=21000 \mathrm{MPa}$. Initial yield stress is $4.1 \mathrm{MPa}$ in the material direction. Initial shear stress is $2.367 \mathrm{MPa}$ and Work hardening parameter is applied to 0. Figure 12, 13 , and 14 show the load-displacement curves for span rise ratio of $0.2,0.3$, and 0.4 and the elastic moduli of Ex=42000MPa and $\mathrm{Ey}=21000 \mathrm{MPa}$.

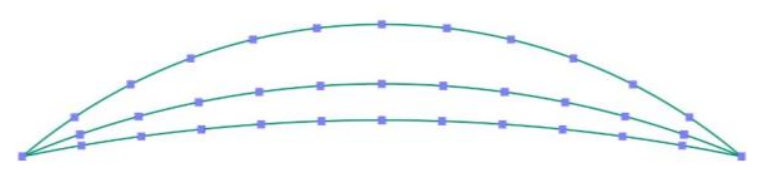

Figure 7. The curve of cylinder 


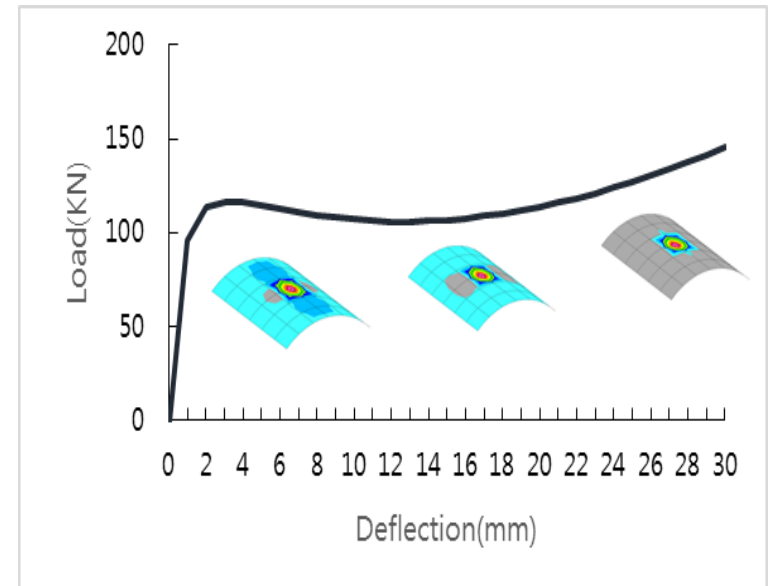

Figure 8. Load displacement curve $($ Span rise ratio $=0.2)$

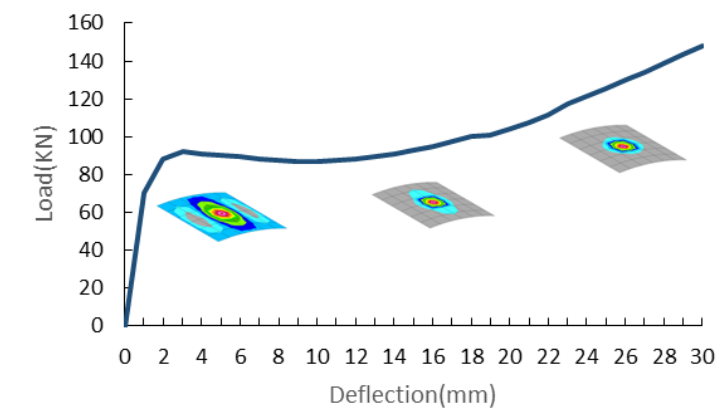

Figure 9. Load displacement curve $($ Span rise ratio $=0.1)$

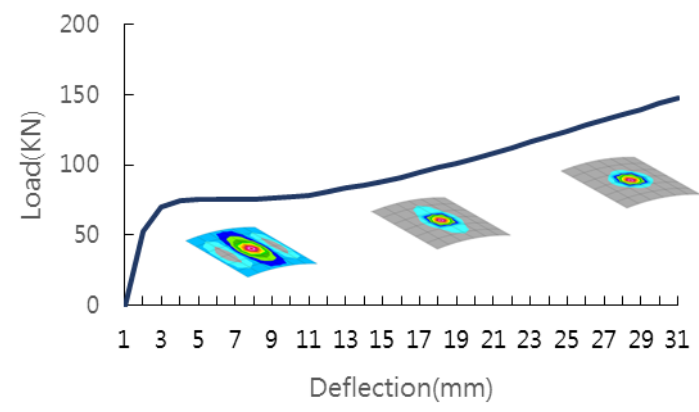

Figure 10. Load displacement curve $($ Span rise ratio $=0.05)$

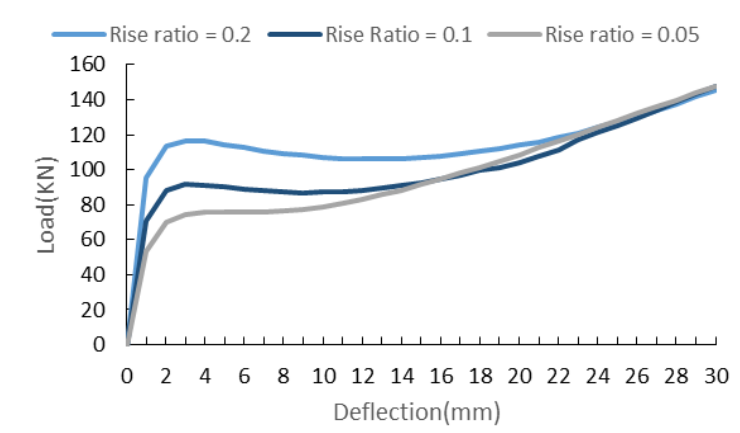

Figure 11. Load displacement curves (Span rise ratio $=0.2,0.1,0.05$ ) 


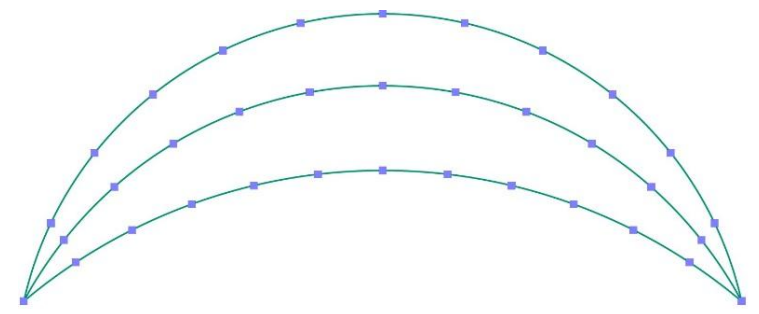

Figure 12. The curve of cylinder

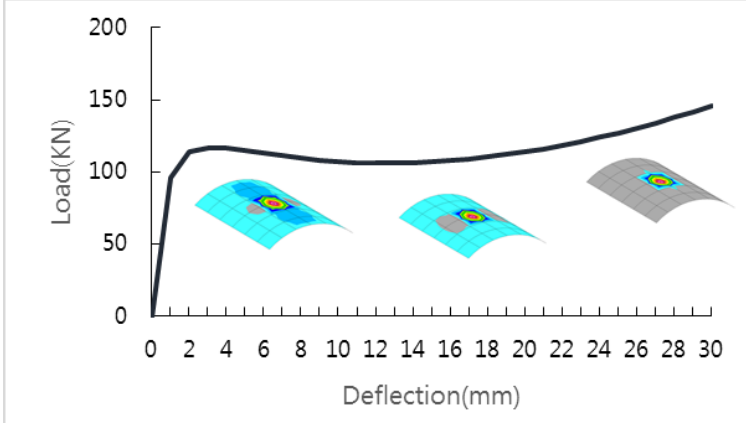

Figure 13. Load displacement curve $($ Span rise ratio $=0.2)$

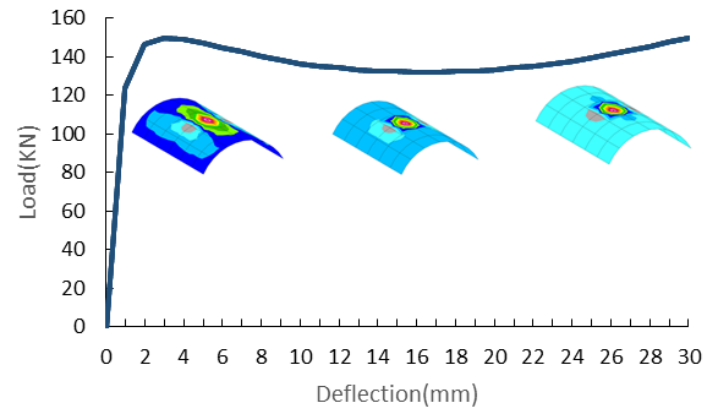

Figure 14. Load displacement curve (Span rise ratio $=0.3)$

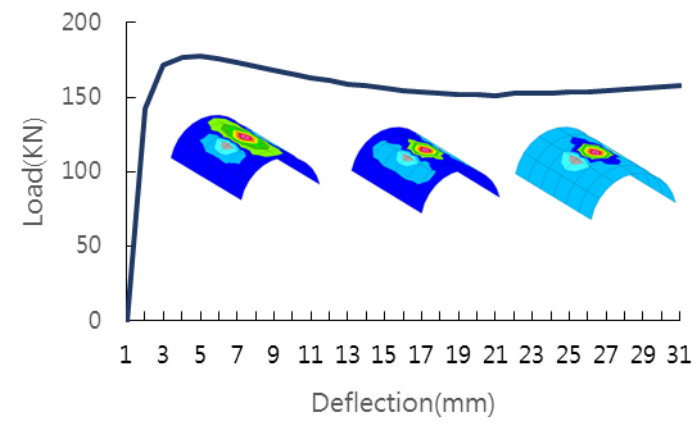

Figure 15. Load displacement curve $($ Span rise ratio $=0.4)$

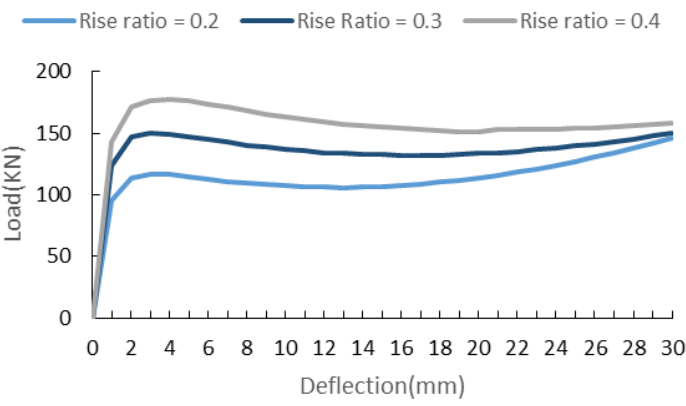

Figure 16. Load displacement curves $($ Span rise ratio $=0.2,0.3,0.4)$ 


\section{IV.CONCLUSION}

In this study, a nonlinear analysis of cylindrical shell was conducted considering the combined geometric and material nonlinearities to analyze the plastic behavior of materials. The shallow shells with a low span rise ratio have a snap back and through behavior for a load condition or a support condition.

(a) The geometric nonlinear analysis of a shallow cylindrical shell under a concentrated load did not revealed the snap-through phenomenon, but the span rise ratio had greatly an affect the load bearing capacity.

(b) In the combined geometric and material nonlinear analysis, after reaching the initial maximum load, the load hardly increased and only deformation continued. When only the material nonlinearity was considered, the load-displacement curve can be identified until the location of maximum load, and the displacement only is increased with no increase in the load.

(c) The material behaved elastically until a certain stress limit, in plastic region the combined geometric nonlinear and plastic behavior was occurred.

\section{ACKNOWLEDGEMENTS}

This research was supported by a grant (17AUDP-B100343-03) from Architecture \& Urban Development Research Program funded by Ministry of Land, Infrastructure and Transport of Korean government.

\section{REFERENCES}

[1] S.Y. Jang, B.J. Son, H.S. J, “A Study on Edge Reinforcement Effect of Cylindrical Shells with Composite Laminate”, Journal of the Korea Society for Advanced Composite Structures, Vol.3, No.4, pp47-54, June 2012.

[2] NISA Software, NISA II User's Manual, Cranes Software International Ltd, 2005.

[3] MIDAS Information Technology Co. Ltd, http://kor.midasuser.com/civil/tech_paper/keyword_view.asp?pg=12\&sk=\&bid=70\&nCat=\&nIndex=\&sHtml=\&idx=748, www.midasit.com

[4] M. A. Cridfied, "Non-linear Finite Element Analysis of Solids and Structures", JOHN WILEY \& SONS Ltd, USA, 1999, 492p, ISBN 047195649X.

[5] Y.S. Kim, "mechanics of plastic deformation and application", Sigmapress Inc, Republic of Korea, 2014, 694p, ISBN 978-89-6866-144-0.

[6] S.E. Han, K.S. Lee, "A Study on The Nonlinear Elasto-Plastic Post Buckling Analysis of Spatial Structures", Journal Architectural Institute of Korea, vol.12, pp.59-68, 2007.

[7] S.E. Han, M.J. KIM, N.W. Kang, "Dynamic Buckling Analysis of Lattice Dome Structures Considering Geometrical Nonlinearity", Journal A rchitectural Institute of Korea, vol.3, pp.3-10, 2009 